\title{
Cisplatin-induced CCL5 secretion from CAFs promotes cisplatin-resistance in ovarian cancer via regulation of the STAT3 and PI3K/Akt signaling pathways
}

\author{
BO ZHOU, CHAOYANG SUN, NA LI, WANYING SHAN, HAO LU, LILI GUO, ENSONG GUO, \\ MENG XIA, DANHUI WENG, LI MENG, JUNBO HU, DING MA and GANG CHEN \\ Cancer Biology Research Center, Tongji Hospital, Tongji Medical College, \\ Huazhong University of Science and Technology, Wuhan, Hubei 430030, P.R. China
}

Received October 27, 2015; Accepted December 4, 2015

DOI: $10.3892 /$ ijo.2016.3442

\begin{abstract}
Currently, acquired resistance to cisplatin (DDP) is a substantial obstacle to reducing the morbidity and mortality due to ovarian malignant tumors. Nevertheless, cisplatin plays a vital role in killing the tumor cells while it may also be a 'primer' involved in chemotherapy resistance. We found that the cisplatin-induced chemokine (C-C motif) ligand 5 (CCL5) secretion derived from cancer-associated fibroblasts (CAFs) promoted ovarian cancer cell resistance to cisplatin. Via a cytokine chip assay, we identified a spectrum of secreted proteins that were derived from the CAFs through cisplatin-induced treatment. Among these, CCL5 significantly attenuated the cytotoxic effect of cisplatin chemotherapy in vitro and in vivo. Additionally, CCL5 expression was also detected in 62 serous ovarian cancer patient tissue specimens using IHC, and the results demonstrated that chemotherapy resistant patients displayed higher expression of CCL5 than the chemo-sensitive patients $(\mathrm{P}<0.05)$. Mechanistically, we found that CCL5 notably increased STAT3 and Akt phosphorylation levels in ovarian cancer cells. These results indicated that cisplatininduced CCL5 secretion derived from the CAFs may promote cisplatin resistance, which was mediated by regulation of the STAT3 and PI3K/Akt signal pathways.
\end{abstract}

\section{Introduction}

Ovarian cancer is the fourth most lethal cancer among women and the leading cause of gynecological cancer deaths worldwide. Currently, the standard first-line therapy for this malignancy includes aggressive cytoreductive surgery followed

Correspondence to: Dr Gang Chen, Cancer Biology Research Center, Tongji Hospital, Tongji Medical College, Huazhong University of Science and Technology, Wuhan, Hubei 430030, P.R. China

E-mail: gumpc@126.com

Key words: ovarian cancer, cancer-associated fibroblasts, cisplatin resistance, STAT3, p-Akt, cisplatin-induced chemokine (C-C motif) ligand 5 by chemotherapy with paclitaxel, platinum-based agents, or a combination of these treatments. Ovarian cancer patients are commonly initially sensitive to these therapies. However, the 5-year-survival has not improved over the past few decades (1) and most survivors eventually relapse and develop resistance to cisplatin therapy. Progression of a cisplatin-resistant tumor ultimately leads to significant morbidity and mortality. The molecular mechanism of cisplatin-resistance remains largely unknown and is a major impediment to more effective cancer treatment. Traditionally, most cytotoxic agents are selectively targeted to cancers by exploiting differential tumor cell characteristics, such as high proliferation rates, hypoxia and genome instability, resulting in a favorable therapeutic index (2). However, platinum-based therapies also affect stromal cells in the tumor microenvironment such as cancer-associated fibroblasts (CAFs), which can disrupt the normal function and physiology of tissues and organs and contribute to tumor drug-resistance (2). The underlying mechanism of cisplatin as a stimulating factor in the stromal cells resulting in cisplatin resistance has not been clarified in ovarian cancer. The present study was undertaken to illuminate this issue.

CCL5 (also known as RANTES), is a member of the $\mathrm{CC}$-chemokine family and plays a crucial role in chemotherapy resistance, relapse, metastasis and migration in human malignancy (3). Many studies have demonstrated that altered CCL5 expression in patients with breast tumors, melanoma, lung, prostate, cervical and pancreatic cancer is significantly correlated with disease progression, poor prognosis and tumor cell chemotherapy resistance (4-7). CCL5 can be expressed and secreted either by tumors themselves or by the tumor microenvironment stromal cells and substantially promotes the resistance of ovarian cancer cells to cisplatin $(8,9)$. However, the role of CCL5 and the mechanisms underlying cisplatin resistance in ovarian cancers have not yet been fully clarified.

The STAT3 is a member of family of transcription factors that mediates the response to a variety of cytokines, chemokines and growth factors and modulates the transcription of genes involved in the regulation of a large variety of vital functions, primarily including cell survival, metastasis, migration, angiogenesis, chemotherapy resistance and the 
immune response (10-14). STAT3 contributes to oncogenesis in many human cancers, including prostate, breast, nasopharyngeal carcinomas and ovarian cancers $(15,16)$. STAT3 pathway activity has been associated with cisplatin resistance in human ovarian carcinomas (17). Furthermore, a previous study demonstrated that using small molecule inhibitors or interference RNA could rescue the inherent and acquired chemoresistance of ovarian cancer cells (18). Moreover, the STAT3-CCL5 signaling pathway has been shown to play an important role in tamoxifen resistance in human breast cancer cells (3). The PI3K/Akt signaling pathway also plays a lead role in tumor progression and chemotherapy resistance and this signaling pathway can be activated by CCL5 (19).

In the present study, we observed a significant correlation between CCL5, STAT3 and PI3K/Akt signaling pathways in regulating cisplatin-induced cisplatin resistance in ovarian cancer cells. We suggest that cisplatin-induced CCL5 secretion derived from CAFs promotes cisplatin resistance in ovarian cancer.

\section{Materials and methods}

Cell culture. The human ovarian cancer cell line SKOV3 was purchased from the American Type Culture Collection (ATCC; Manassas, VA, USA). A cisplatin-resistant ovarian cancer cell line $\left(\mathrm{C}_{13} *\right)$ was a gift from Professor Benjamin K. Tsang, Ottawa Health Research Institute, Ottawa, Canada (20). Cells were cultured in Macoy'5A medium (Gibco, Carlsbad, CA, USA) supplemented with $10 \%$ fetal bovine serum (FBS; Gibco), penicillin (100 units/ml) and streptomycin $(100 \mu \mathrm{g} / \mathrm{ml})$ at $37^{\circ} \mathrm{C}$ in a humidified atmosphere containing $5 \% \mathrm{CO}_{2}$.

Reagents. Recombinant human CCL5 (RANTES) was purchased from PeproTech, Inc., (Rocky Hill, NJ, USA) and anti-CCL5 neutralizing antibody was purchased from R\&D Systems (Minneapolis, MN, USA). Cisplatin (DDP) was purchased from Sigma-Aldrich (St. Louis, MO, USA). LY294002 was obtained from Selleck Chemicals (Houston, TX, USA).

Patient samples. Sixty-two patients with serous ovarian cancer staged as III-IV (FIGO) at Tongji Hospital (Wuhan, Hubei, China) provided informed consent for the collection of solid tumor specimens during the years 2010 to 2013. All patients underwent debulking and subsequent platinumcentered chemotherapy. The protocol was approved by the Ethics Committee of Tongji Hospital. Platinum resistance or platinum sensitivity was defined as relapsed or progression within six months or after six months from the last platinumbased chemotherapy, respectively.

Isolation and primary culture of fibroblasts and immunocytochemistry (ICC) on cultured CAFs. Fresh tumor tissue samples were obtained from patients who underwent initial cytoreductive surgery diagnosed as high-grade serous ovarian cancer through intraoperative fast biopsy. The detailed procedure was performed as previously described (21). Primary antibodies included $\alpha$-smooth muscle actin ( $\alpha$-SMA) monoclonal antibody (1:100 dilution; Abcam, Cambridge UK); mouse monoclonal antibody vimentin (1:100 dilution; Wuhan
Boster Biological Technology, Ltd., Wuhan, China); mouse monoclonal antibody cytokeratin 8 (1:100 dilution; Wuhan Boster Biological Technology).

Conditioned medium. DDP was administered to CAFs at proper concentrations for $12 \mathrm{~h}$, and CAFs without DPP administration were used as control. The medium was replaced with serum-free medium for culturing for another $24 \mathrm{~h}$. The treated supernatant was referred to as $\mathrm{CM}^{*}$, and the untreated supernatant as the control medium.

siRNA transfection assay. The STAT3 siRNA transfection experiment was performed according to the manufacturer's protocol using Lipofectamine ${ }^{\mathrm{TM}} 3000$ (Invitrogen). The siRNA sequences for STAT3 were 5'-GGA GCA GCA CCU UCA GGA UTT-3' as previously described (18).

CCL5 neutralization assay. Anti-CCL5 antibody neutralization was added to the culture cell using $10 \mu \mathrm{g} / \mathrm{ml}$ with or without recombinant human CCL5.

Drug sensitivity assay. A cell counting kit was used to analyze cell viability. Cells were cultured in 96-well plates overnight $\left(1 \times 10^{4}\right.$ cells/well). After $24 \mathrm{~h}$, cells were treated with DDP at indicated concentrations in $\mathrm{CM}^{*}$ or control medium for $48 \mathrm{~h}$. After addition of CCK- 8 for $2 \mathrm{~h}$, the number of survival cells was detected at the absorbance of $450 \mathrm{~nm}$ by a microplate reader (Bio-Rad Laboratories). Each experiment was performed three times. The results were analyzed using GraphPad Prism 5 (GraphPad Software Inc., La Jolla, CA, USA).

Apoptosis assay. Apoptosis assay was performed as previously described (22).

Western blot analysis. Western blot analysis was performed as previously described (22). Primary mouse monoclonal antibody against human MRP1 and MRP2 (1:1,000 dilution; Wuhan Boster Biological Technology), rabbit polyclonal antibody against human Bcl-2 (1:1,000 dilution; Epitomics, Burlingame, CA, USA), rabbit polyclonal antibody against human cleaved-PARP (1:1,000 dilution; Epitomics), rabbit monoclonal antibody against human activated caspase-3 (1:1,000 dilution; Cell Signaling Technology, Inc., Beverly, MA, USA) and p-Akt ${ }^{\text {ser473 }}$ (1:1,000 dilution; Cell Signaling Technology), and goat monoclonal antibody against human CCL5 (1:1,000 dilution; R\&D Systems, Inc., Minneapolis, MN, USA), and rabbit monoclonal antibody against human p-STAT3 (Tyr705, 1:1,000 dilution; Abcam).

Cytokine/chemokine array. CAFs were first pretreated with DDP at a concentration of $10 \mu \mathrm{M}$ or without DDP for $12 \mathrm{~h}$, and then replaced with serum-free medium for another $24 \mathrm{~h}$. Next, the conditioned medium (defined as $\mathrm{CM}^{*}$ ) and control medium were centrifuged and the supernatant was passed through a $0.22 \mu \mathrm{m}$ filter. The $\mathrm{CM}^{*}$ and control medium were tested using the RayBio Human Cytokine Antibody Array G Series 5 according to manufacturer's instructions. After blocking the array chip, $100 \mathrm{ml}$ of sample was added per sub-array for incubation. Subsequent washes and biotin-conjugated antibody and 
Table I. Primers for real-time RT-PCR.

\begin{tabular}{lll}
\hline Gene & \multicolumn{1}{c}{ Sense } & \multicolumn{1}{c}{ Antisense } \\
\hline GAPDH & 5'-GAAATCCCATCACCATCTTCCAGG-3' & 5'-GAGCCCCAGCCTTCTCCATG-3' \\
CCL5 & 5'-CTTGACCTGTGGACGACTGC-3' & 5'-ATCCAGTGAGAAAAGCCCGT-3' \\
MIF & 5'-CCGGACAGGGTCTACATCAA-3' & 5'-GCGAAGGTGGAGTTGTTCCA-3' \\
MIP-1ß & 5'-AGCACCAATGGGCTCAGAC-3' & 5'-TCACTGGGATCAGCACAGAC-3' \\
MCP-3 & 5'-TGCTCAGCCAGTTGGGATTA-3' & 5'-GTGGCTACTGGTGGTCCTTC-3' \\
IL-6 & 5'-ACCCCCAATAAATATAGGACTGGA-3' & 5'-AAGGCGCTTGTGGAGAAGG-3' \\
IL-8 & 5'-TGTGAAGGTGCAGTTTTGCCA-3' & 5'-ACCCAGTTTTCCTTGGGGTC-3' \\
OPG & 5'-CATGTTCGTGGCCCTCCTG-3' & 5'-GGATCCATCTGCGCTCTGAA-3' \\
STAT3 & 5'-ACCTGCAGCAA-TACCATTGAC-3' & 5'-AAGGTGAGGGACTCAAACTGC-3' \\
\hline
\end{tabular}

OPG, osteoprotegerin; CCL5 is also known as RANTES.

fluorescent dye-conjugated streptavidin incubations followed, and fluorescence detection was achieved using a 4000A Axon GenePix laser scanner. Background-deducted signal values were used and normalized against positive controls within the chip. Data are expressed as a ratio of $\mathrm{CM}^{*}$ to control media.

RNA extraction and real-time RT-PCR. Total RNA was extracted from CAFs or cell lines using the PrimeScript RT reagent kit (Takara) and SYBR Premix Ex Taq (Takara) according to the manufacturer's instructions. Primer sequences for the endogenous reference genes, glyceraldehyde-3-phosphate dehydrogenase (GAPDH), and the specific primer sequences used are shown in Table I. The comparative $\mathrm{Ct}$ method was used to calculate the relative changes in gene expression.

Colony formation assay. Chemosensitivity was also determined by a standard colony formation assay. Briefly, cells seeded in 6-well plates were treated with or without continuous CCL5 (100 ng/ml) at indicated doses and then exposed to DDP $(50 \mu \mathrm{mol} / \mathrm{l})$. Plates were incubated at $37^{\circ} \mathrm{C}$ for 14 days then stained with crystal violet and counted. Each assay was performed in triplicate.

Paraffin section immunohistochemistry and immunofluorescence. Immunohistochemistry and immunofluoresence staining were done as previously described (23). The results for CCL5 staining were scored on the basis of the cell cytoplasm staining (score 0, no cytoplasm staining; score 1, weak staining; score 2, moderate staining; score 3, strong staining). For further statistical analysis, scores 0 and 1 were categorized as low expression, and scores 2 and 3 were categorized as high expression as previously described.

In vivo xenograft studies. Twelve female nude BALB/c mice (Beijing HFK Bioscience Co., Ltd., Beijing, China) were subcutaneously injected $5 \times 0^{6} \mathrm{C} 13^{*}$ cells resuspended in $100 \mu \mathrm{l}$ phosphate-buffered saline into the left flank and $5 \times 10^{6} \mathrm{C} 13^{*}$ cells mixed with $2.5 \times 10^{6} \mathrm{CAF}$ cells injected into the right flank. Mice were housed under specific pathogen-free conditions and all animal experiments were carried out in accordance with the Guide for the Care and Use of Laboratory Animals of Tongji Hospital in Wuhan, China. When tumors reached a mean size of $50 \mathrm{~mm}^{3}$, the mice were randomly assigned into two groups with 6 mice per group. The experiment group was treated with DDP $5 \mathrm{mg} / \mathrm{kg}$ intraperitoneally once a week for four weeks. The control group mice were injected with sterile saline. Tumor volumes were calculated as length $\mathrm{x}$ width ${ }^{2} / 2$. The tumors were weighed at sacrifice following cervical dislocation under anesthesia.

Statistical analysis. All data are expressed as mean \pm SD. The means were calculated at least from three independent experiments. Statistical significance of differences was analyzed by two-tailed Student's t-test or ANOVA. P-value $<0.05$ was considered statistically significant. The statistical analyses were done using the SPSS 17.0 (SPSS Inc., Chicago, IL, USA).

\section{Results}

DDP-induced cytokine secretion derived from CAFs promotes ovarian cancer cell resistance to DDP in vitro. A previous study indicated that chemotherapy-induced cytokine or chemokine secretion from the tumor microenvironment promoted prostate cancer therapy resistance (2). We first obtained primary human CAFs isolated from patients diagnosed with highgrade serous ovarian cancer undergoing initial cytoreductive surgery. Morphological and immunocytochemical evaluation showed that CAFs displayed no cytokeratin 8 staining, but strongly expressed vimentin and $\alpha$-SMA, the makers of mesenchymal cells (Fig. 1A). To verify whether DDP contributes to the cytokine secretion of the tumor microenvironment while killing tumor cells, which results in the cisplatin resistance of the ovarian cancer, we administered DDP to C13* and SKOV3 cells at the proper concentration for $48 \mathrm{~h}$ in $\mathrm{CM}^{*}$ or control conditional medium, and CCK8 was used to detect C13* or SKOV3 cell viability. Ovarian cancer cells were more resistant to DDP in $\mathrm{CM}^{*}$ than control medium (Fig. 1B and C). The apoptosis rates of $\mathrm{C}^{*} 3^{*}$ or SKOV3 cells were also calculated by flow cytometric analysis (Fig. 1D and E). Western blotting assay indicated that the anti-apoptosis protein $\mathrm{Bcl}-2$ was increased and that the apoptosis proteins cleaved-PARP 
A
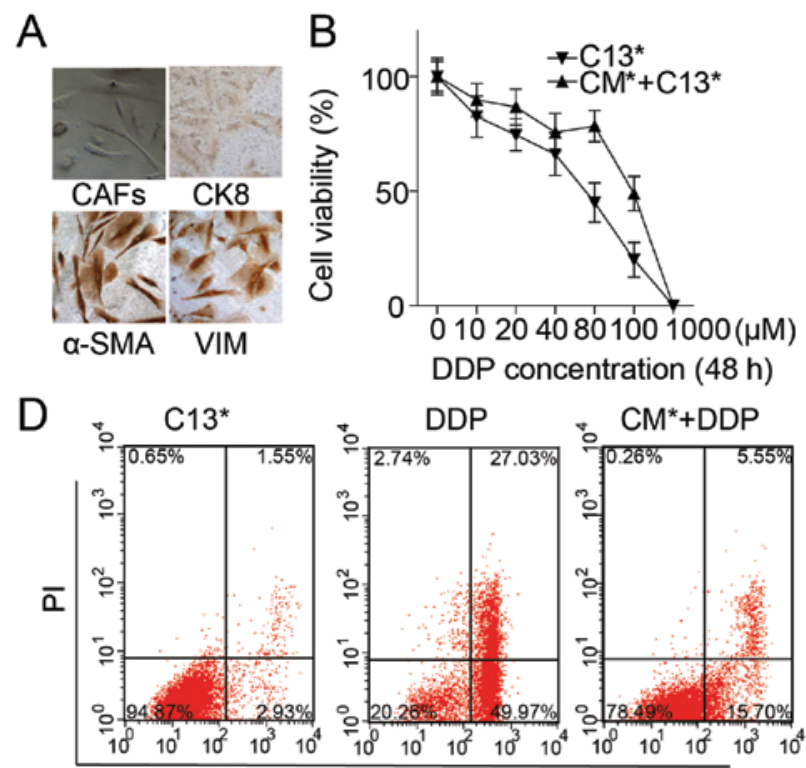

$\mathrm{E}$

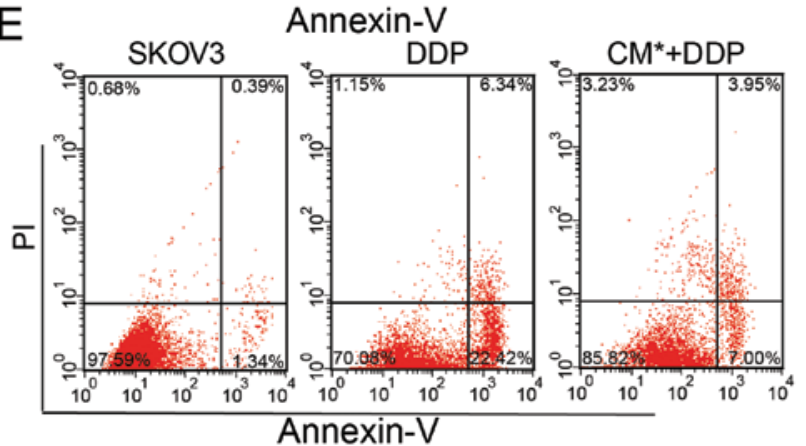

$\mathrm{F}$

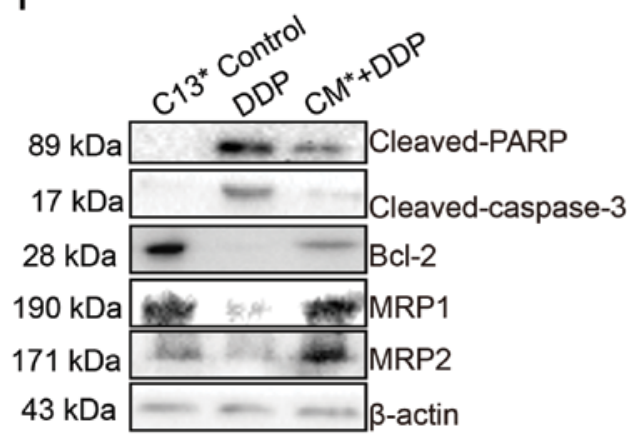

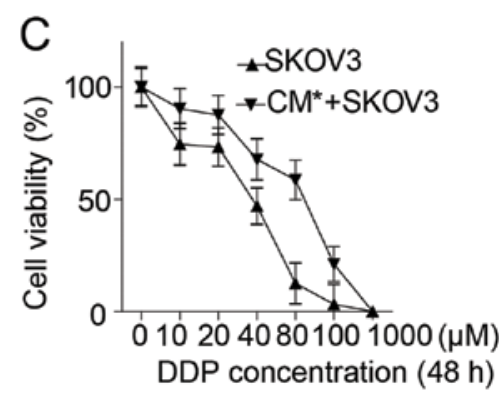
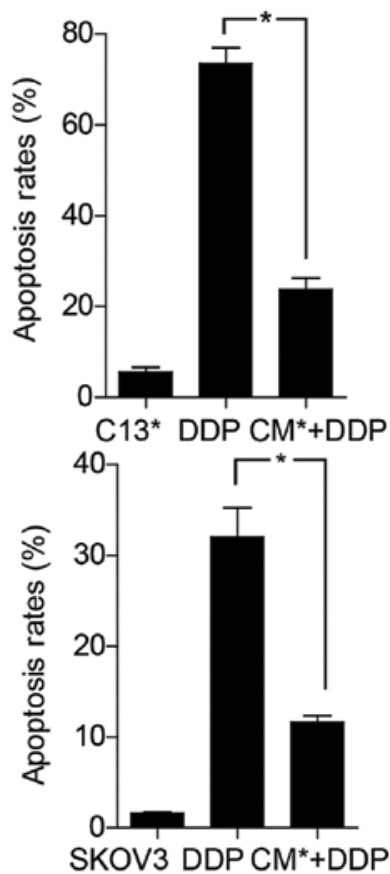

G

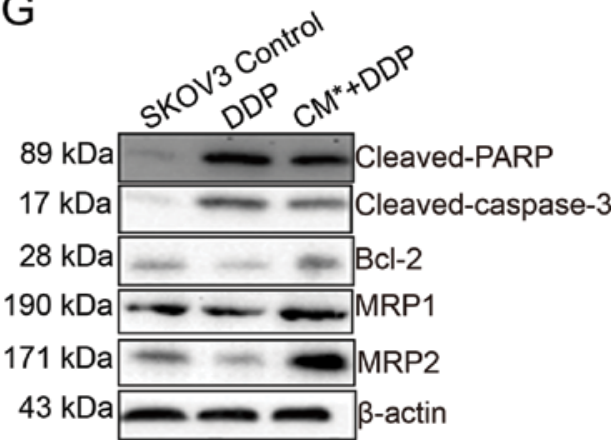

Figure 1. DDP induced cytokine secretion derived from CAFs promotes ovarian cancer cell resistance to DDP in vitro. (A) Characterization of CAFs, which stain negative for CK8 (cytokeratin 8), positive for VIM (vimentin) and $\alpha$-SMA ( $\alpha$-smooth muscle actin). (B and C) C13* and SKOV3 cells displayed more resistance to DDP in $\mathrm{CM}^{*}$ than control medium, cell viability was performed after treatment with a range of concentrations of DDP for $48 \mathrm{~h}$ via CCK-8. (D and E) The apoptosis rates of C13* and SKOV3 cells were detected by flow cytometry after DDP treatment in control medium or in CM*. (F and G) Expression level of apoptosis-associated proteins or drug-resistance-associated proteins were reduced or increased, respectively, though western blot assay.

and cleaved caspase- 3 were markedly decreased. MRP1/2 (multidrug-resistance proteins $1 / 2$ ) is a major protein involved in MDR (multidrug resistance) (24-26). MDR has a profound effect on cancer chemotherapy. In contrast, MRP1 and MRP2 were significantly increased compared with control (Fig. 1F and G).

CCL5 is strongly secreted from CAFs after cisplatin treatment. To determine which factors influence acquired cisplatin resistance after cisplatin treatment, cytokine/chemokine assay was performed according to the manufacturer's protocol. As shown in Fig. 2A and B, a series of factors were secreted and increased in $\mathrm{CM}^{*}$ compared with the control medium. Many of these factors were associated with tumor cell malignant phenotype, including chemotherapy resistance and tumor progression, migration and invasion (27-29). CCL5 was significantly increased up to $\sim 72$-fold compared with the control medium. We also detected these cytokines with qRT-PCR and found that CCL5 expression was increased up to 12-fold compared with control (Fig. 2C). A previous study demon- 


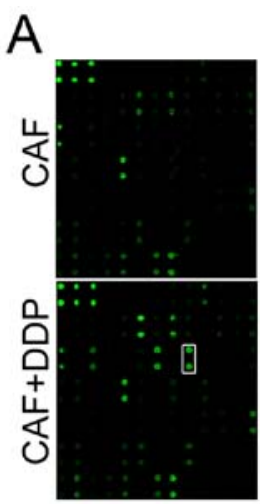

B
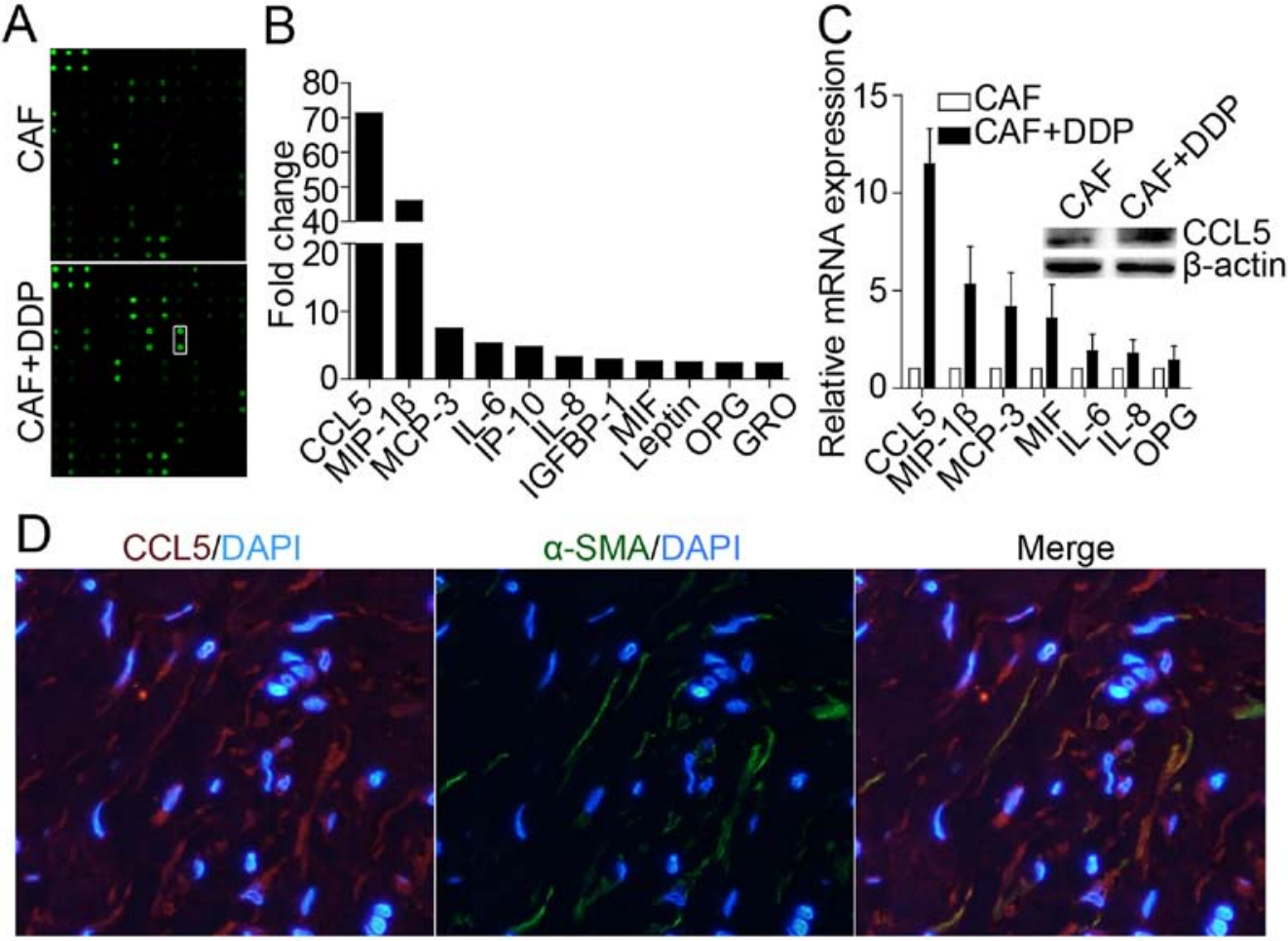

\section{$\alpha-S M A / D A P \mid$}

\section{Merge}

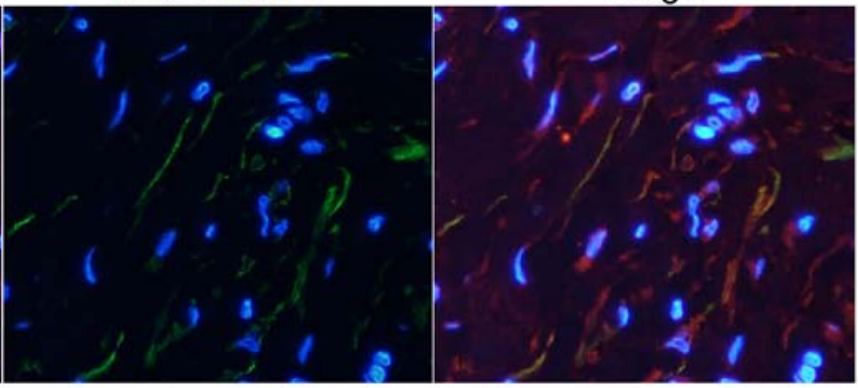

Figure 2. CCL5 is strongly secreted from CAFs after DDP treatment. (A and B) Cytokine chip was used to screen high express and specific cytokines, thus CCL5 increased up to 72 -fold in $\mathrm{CM}^{*}$ compared with that in control medium. (C) CAFs were treated with or without indicated concentrations of DDP for $12 \mathrm{~h}$ and then replaced with serum-free medium for culturing for another $24 \mathrm{~h}$. qRT-PCR and western blot analysis were used to detect related cytokines and the results showed that CCL5 (RANTES) was highly expressed in CAFs treated with DDP than without DDP treatment. (D) Paraffin section immunofluorescence stain was used to locate CCL5 and CAFs. The high grade serous ovarian cancer tissue sections were counterstained with DAPI (blue signal), $\alpha$-SMA positive stromal cells co-expressed CCL5 (red signal), suggesting that CAF cells may produce CCL5. Data represent the means \pm standard deviation. ${ }^{*} \mathrm{P}<0.05$. Each experiment was repeated in triplicate.

strated that CCL5 could be secreted or expressed by tumor cells or by extracellular matrix (ECM) $(4,6,7,9)$. We found that CCL5 was derived from the tumor microenvironment of CAFs, which was further confirmed through immunofluorescence double staining co-localization, $\alpha$-SMA-positive stromal cells co-expressed CCL5, suggesting that CAF cells may produce CCL5 (Fig. 2D).

CCL5 stimulation enhanced ovarian cancer cell resistance to cisplatin. To verify the function of CCL5 in ovarian cancer cells, Annexin V/PI double staining assay was used to assess C13* or SKOV3 cell apoptosis. CCL5 stimulation significantly decreased tumor cell apoptosis rates compared with cisplatin treatment alone (Fig. 3A and C). In addition, C13* and SKOV3 cells were selected to determine cell viability via CCK- 8 after treatment with or without CCL5 stimulation for $48 \mathrm{~h}$. $\mathrm{C}_{13}{ }^{*}$ and SKOV3 cells were more resistant to cisplatin after CCL5 treatment compared with C13* and SKOV3 cells not undergoing CCL5 treatment (Fig. 3D and E). Moreover, C13* cells treated with CCL5 $(100 \mathrm{ng} / \mathrm{ml})$ exhibited $\sim 20 \%$ increased colony formation compared with control after exposure to DDP (50 $\mu \mathrm{mol} / \mathrm{l})$ (Fig. 3F). These results support that CCL5 stimulation enhances cisplatin resistance.

CCL5 stimulation promotes cisplatin resistance of ovarian cancer cells via the p-STAT3 and PI3K-Akt signaling pathways in vitro and in vivo. Previous studies have demonstrated that constitutively activated phosphorylation of STAT3 contributed to the development of tumor growth, drug resistance and angiogenesis (30). The PI3K/Akt signaling pathway plays a vital role in the regulation of numerous cellular functions, including tumor progression, angiogenesis, adhesion, migration, survival and drug resistance, in many human cancers (31) and is involved in cisplatin-based chemotherapy resistance in epithelial ovarian cancer (32). In addition, the Akt signaling pathway can be activated via chemokines or cytokines, such as CCL5 $(19,33,34)$. We hypothesized that these two pathways were involved in the regulation of cisplatin resistance by CCL5. We treated C13* and SKOV3 cells with a range of CCL5 concentrations for different durations. Phosphorylation of STAT3 (Tyr705) and Akt (ser473) was increased in a time- and dose-dependent manner, as indicated by western blot assay (Fig. 4A and B). Then, $100 \mathrm{ng} / \mathrm{ml} \mathrm{CCL5}$ with or without DDP was administered for $48 \mathrm{~h}$ because this concentration and duration resulted in the highest STAT3 and Akt phosphorylation. Western blot assay demonstrated that p-STAT3 and p-Akt expression was strongly enhanced when cells were treated with DDP combined with CCL5 compared with that with DDP alone (Fig. 4C and D). Expression of the anti-apoptosis protein $\mathrm{Bcl}-2$ was increased, and expression of the apoptosis proteins cleaved PARP and cleaved caspase-3 was decreased (Fig. 4C and D). We next examined the effect of anti-CCL5 antibody on the apoptosis rate of $\mathrm{C13}^{*}$ and SKOV 3 cells. Flow cytometry indicated that the apoptosis rate was increased via concomitant administration of anti-CCL5 neutralization antibody (Fig. 4E and F), and p-STAT3 and 

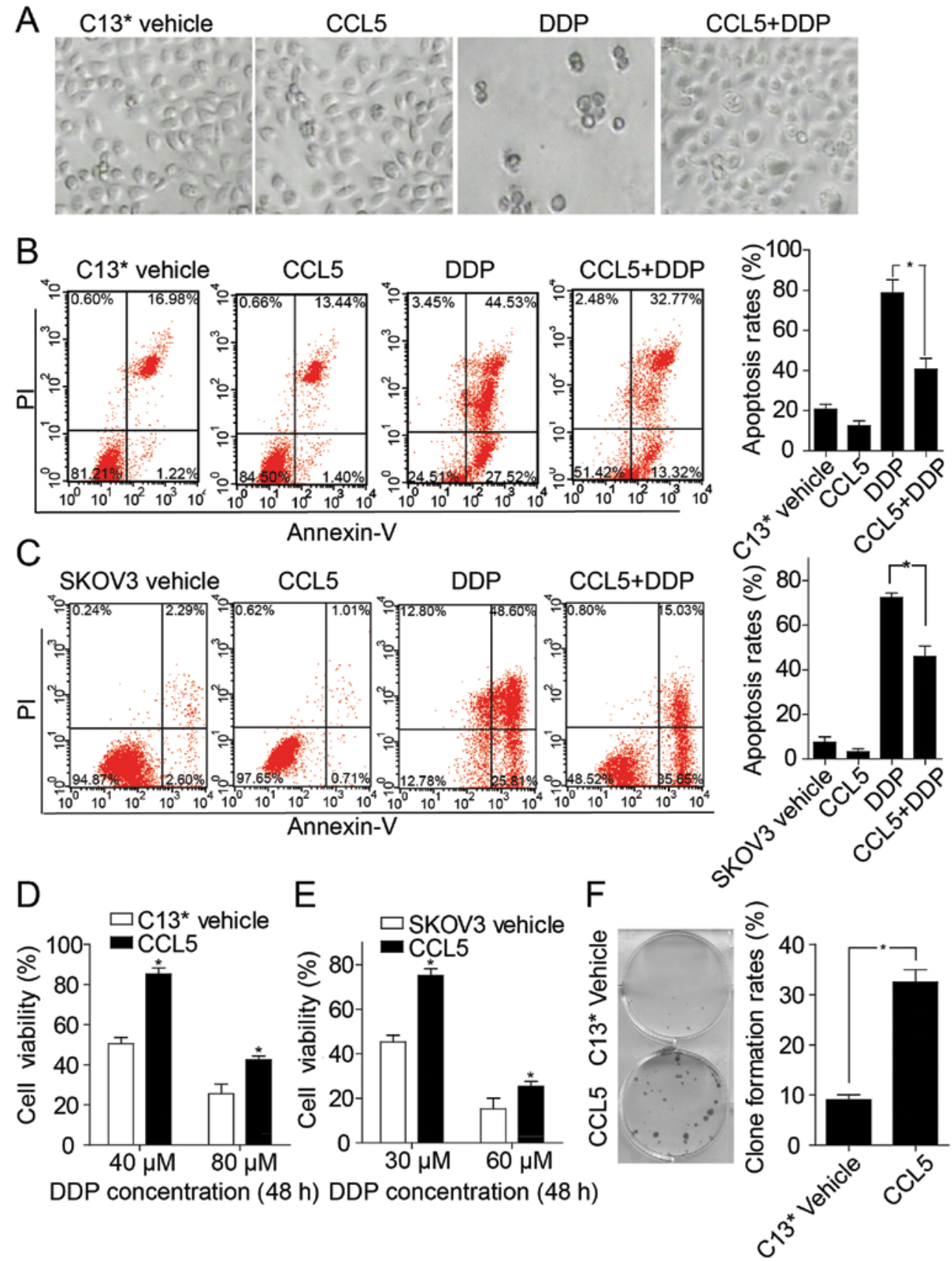

Figure 3. CCL5 promotes ovarian cancer cell resistance to DDP. (A) C13* and SKOV3 cells were treated with CCL5 (100 ng/ml) with or without DDP at indicated concentrations, which were morphologically assessed by a Leica microscope (SKOV3 data not shown). (B and C) CCL5 weakened DDP induced apoptosis rates of $\mathrm{C} 13^{*}$ and SKOV3 cells by FITC. (D and E) C13* and SKOV3 cells were treated with recombination human CCL5 (100 ng/ml) and then treated with an increasing concentration of DDP for $48 \mathrm{~h}$. Cell viability was detected using CCK-8 method. (F) Colony formation of C13* cells was evaluated with or without recombination human CCL5 $(100 \mathrm{ng} / \mathrm{ml})$ every 3 days and then exposed to DDP $(50 \mu \mathrm{mol} / \mathrm{l})$ for 2 weeks. Data represent the means \pm standard deviation. ${ }^{*} \mathrm{P}<0.05$. Each experiment was repeated in triplicate.

p-Akt levels were decreased, as indicated by western blot assay (data not shown). To further confirm whether the STAT3 and Akt signaling pathways are involved in the regulation of cisplatin resistance via CCL5, small interfering RNA for STAT3 and small molecular inhibitor LY294002 for inhibiting Akt were used. CCL5-induced anti-apoptosis was abolished by STAT3 siRNA and the PI3K-Akt inhibitor LY294002, as indicated using flow cytometry Annexin V/PI (Fig. 4G and H). In contrast, CCL5-induced phosphorylation levels of STAT3 and Akt were decreased by STAT3 siRNA and LY294002 treatment (data not shown). These results suggest that CCL5 stimulation enhanced cisplatin resistance via the STAT3 and PI3K/Akt signaling pathways.

Expression and clinicopathological characteristics of CCL5 in ovarian cancer patients. Previous studies have demonstrated that high tissue and plasma CCL5 levels are correlated with advanced breast cancer and could be used as a prognostic indicator for breast, cervical and gastric cancers $(6,35,36)$. Serum CCL5 may be useful in differentiating benign ovarian tumors from malignancy (37). We examined ovarian cancer tissue excised from a patient prior to chemotherapy and 
A

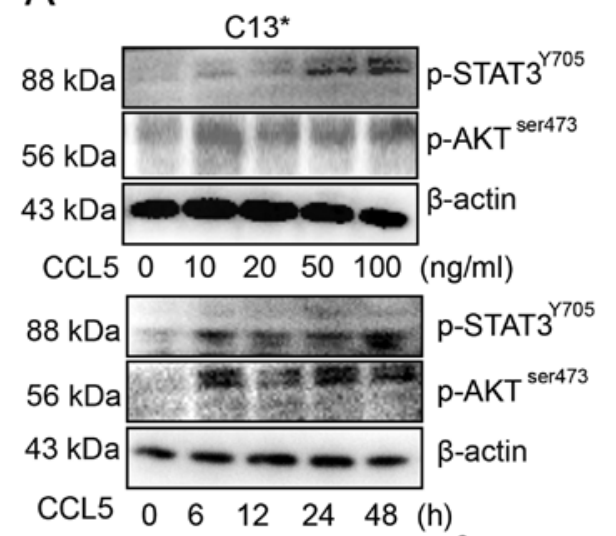

C

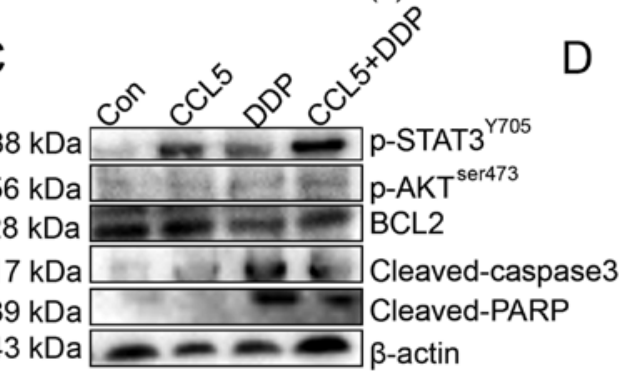

$\mathrm{C} 13^{\star}$

$\mathrm{E}$

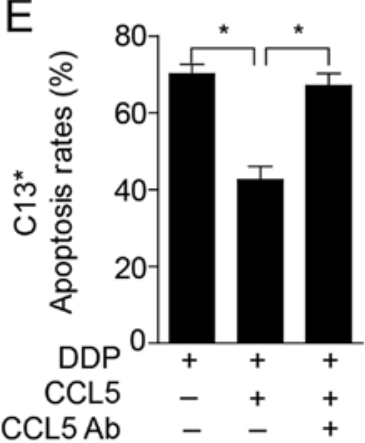

$\mathrm{G}$

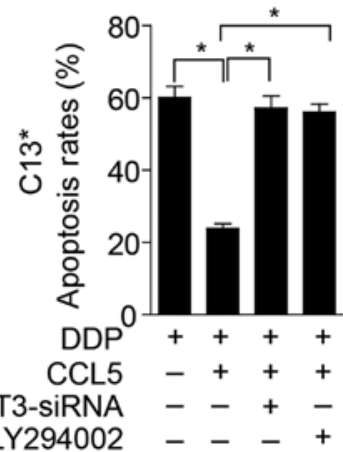

B

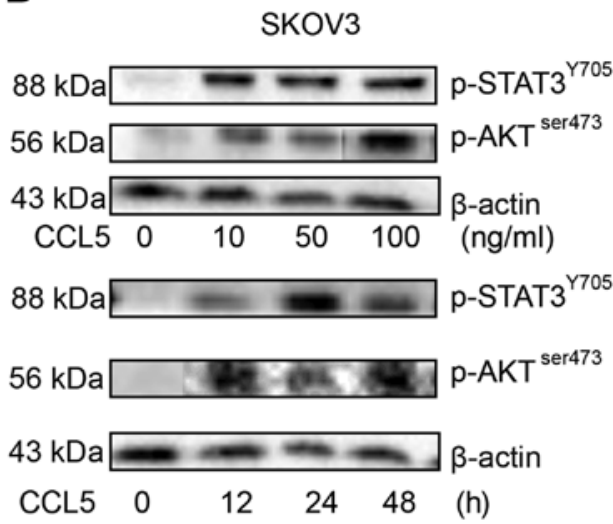

D

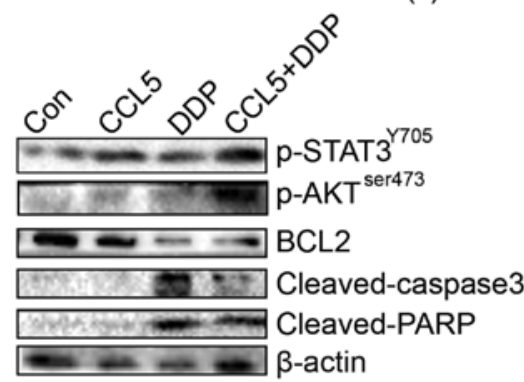

SKOV3

$\mathrm{F}$

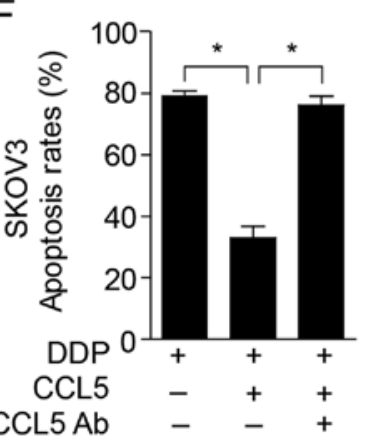

$\mathrm{H}$

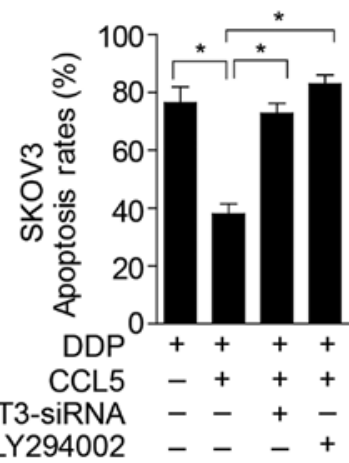

Figure 4. CCL5 promotes DDP resistance of ovarian cancer cells via activating phosphorylation of the STAT3 and AKT signaling pathways. (A and B) C13* and SKOV3 cells were treated with a range of CCL5 concentrations for different durations and concentrations. Phosphorylation of STAT3 (Tyr705) and Akt (ser473) was increased in a time- and dose-dependent manner assayed by western blot analysis. (C and D) C13* and SKOV3 cells were treated with DDP, CCL5 or combination of both for $48 \mathrm{~h}$, then western blot analysis was used to detect the apoptosis-related proteins levels and the levels of phosphorylation of STAT3 and AKT. (E and F) Effect of CCL5 neutralization treatment. C13* and SKOV3 cells were treated with DDP, CCL5, CCL5 antibody (10 $\mu$ g/ml) and their combination for $48 \mathrm{~h}$, then the apoptosis rates were detected by FITC. (G and H) Effect of STAT3 siRNA or small molecular inhibitor LY294002 treatment. C13* and SKOV3 cells were first transfected with STAT3 siRNA for $24 \mathrm{~h}$, then treated with DDP, CCL5, LY294002 (20 $\mu \mathrm{M})$ or combination of both for another 48 h. Data represent the means \pm standard deviation. ${ }^{*} \mathrm{P}<0.05$. Each experiment was repeated in triplicate.

relapsed tissues after four courses of standard chemotherapy, CCL5 expression was significantly increased after exposure to chemotherapy, as demonstrated by IHC (Fig. 5A). Moreover, we assessed CCL5 expression in 62 serous ovarian cancer patient tissue sections using IHC. CCL5 was strongly expressed and secreted both by tumor cells and the tumor stromal cells. Clinic data demonstrated that platinum-resistant patients had higher CCL5 expression than platinum-sensitive patients (Fig. 5B 


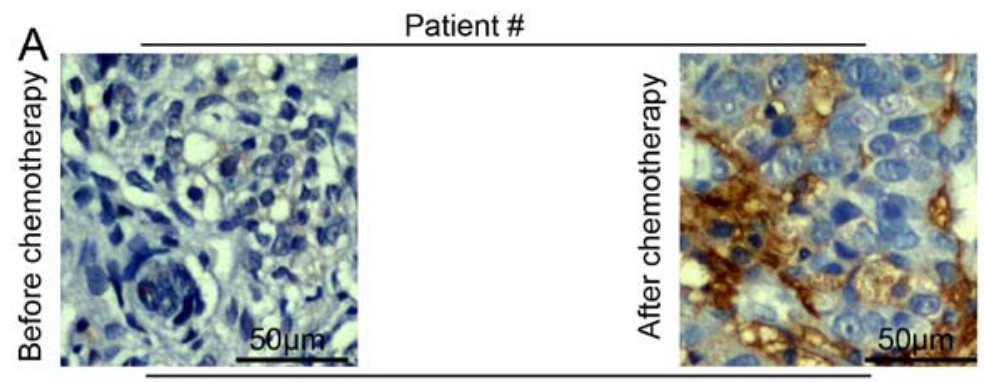

CCL5 expression

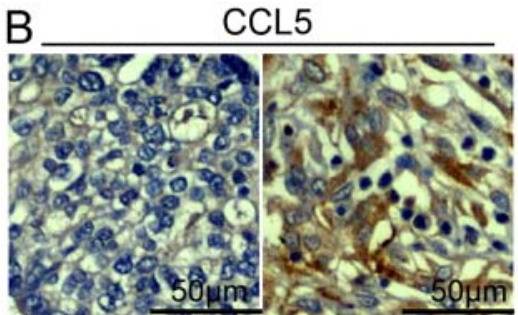

Sensitive

Resistant

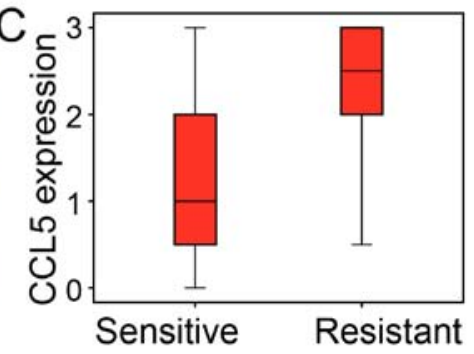

Figure 5. Clinical roles of CCL5 expression in late-stage ovarian cancer patients. (A) CCL5 expression of a patient tissue from before chemotherapy and after chemotherapy detected by IHC. CCL5 displayed strongly positive expression after chemotherapy. (B) CCL5 displayed significantly higher expression in ovarian cancer patients resistant to DDP treatment compared with in ovarian cancer patients sensitive to DDP treatment analyzed by IHC. (C) CCL5 expression was higher in platinum-resistant patients than platinum-sensitive patients.
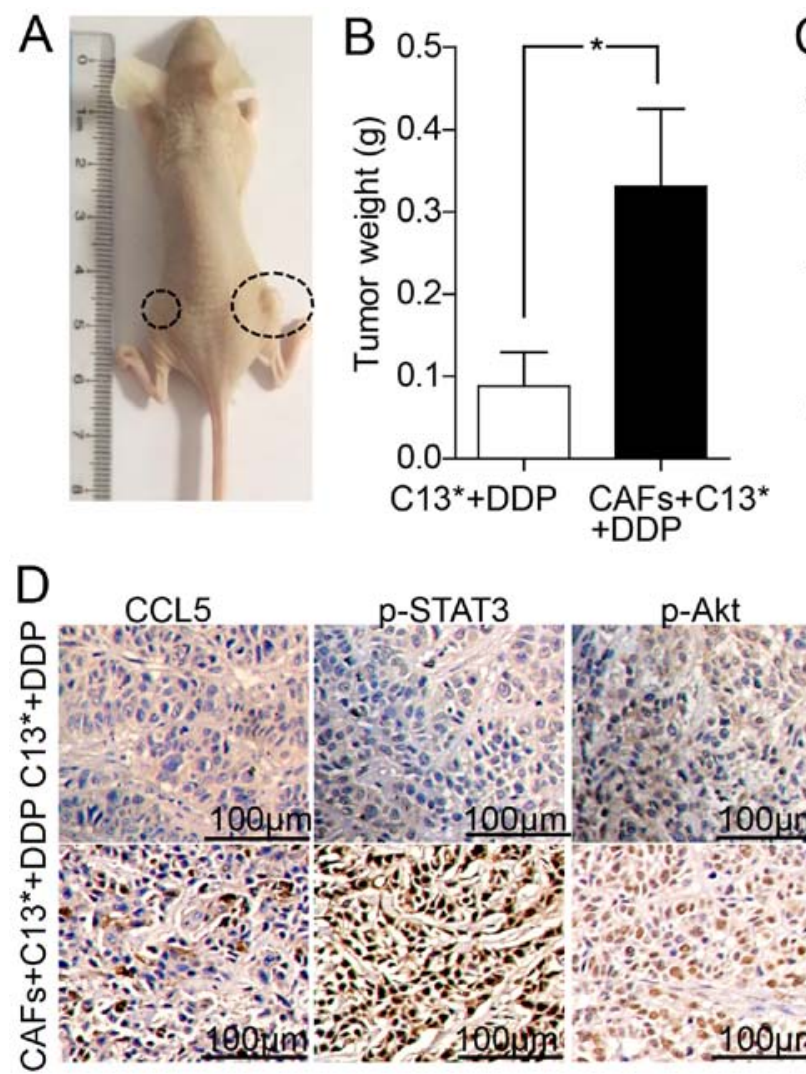

Figure 6. CCL5 derived from CAFs promotes ovarian cancer cell resistance to DDP in vivo. (A-C) C13* cells alone and C13* cells with CAFs were subcutaneous injected into the left and the right flank, respectively. Tumor volumes were calculated and the tumor weight was measured as described in Materials and methods. Arrows represented the start and the end of DDP treatment, $\mathrm{n}=6$ per group. (D) CCL5, p-STAT3, p-Akt and Ki-67 proteins were significantly increased in the right flank tumors compared with the left flank tumors after DDP treatment, whereas activated caspase-3 was decreased. Data represent the means \pm standard deviation. ${ }^{*} \mathrm{P}<0.05,{ }^{* * *} \mathrm{P}<0.01$.

and C) $(\mathrm{P}<0.0001)$. Moreover, CCL5 expression was also positively associated with cancer stage (Table II) $(\mathrm{P}<0.0001)$.

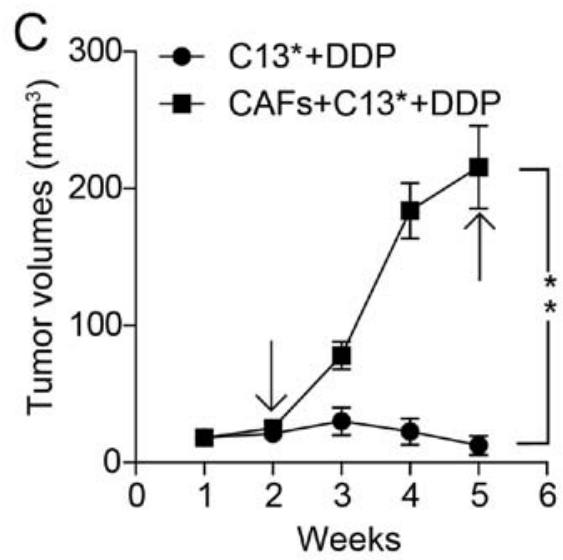

Ki-67 activated caspase3

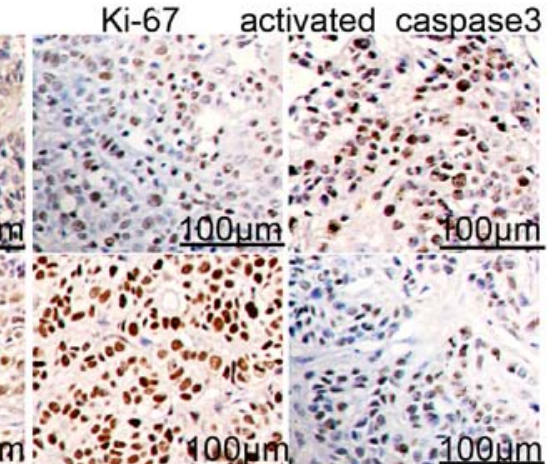


Table II. Correlation of CCL5 expression with clinicopathological characteristics of late-stage ovarian cancer patients (N=62).

\begin{tabular}{lccc}
\hline & & & CCL5 \\
\cline { 3 - 3 } Variables & $\mathrm{N}$ & Low $(\mathrm{n}=24) \mathrm{n}(\%)$ & High $(\mathrm{n}=38) \mathrm{n}(\%)$ \\
\hline Age (years) median (range) & $52(38-72)$ & & $1(2.6)$ \\
Differentiation & & & $12(31.6)$ \\
Well & 3 & $2(8.3)$ & $25(65.8)$ \\
Moderate & 20 & $8(33.3)$ & $11(28.9)$ \\
Poor & 39 & $14(58.4)$ & $27(71.1)$ \\
Lymph node metastasis & & $4(16.7)$ & 0.568 \\
Positive & 15 & $20(83.3)$ & $30(78.9)$ \\
Negative & 47 & & $8(21.1)$ \\
FIGO stage & & $21(87.5)$ & $16(42.1)$ \\
IIIa-IIIc & 51 & $3(12.5)$ & $22(57.9)$ \\
IV & 11 & $21(87.5)$ & $<0.0001^{\mathrm{a}}$ \\
Chemotherapy & & $3(12.5)$ & \\
Sensitive & 37 & & $<0.0001^{\mathrm{a}}$ \\
Resistant & 25 & &
\end{tabular}

ap-values $<0.05$.

and lymph node metastasis. These data suggest that CCL5 is significantly associated with platinum-based chemotherapy response.

CCL5 derived from CAFs promotes ovarian cancer cell resistance to $D D P$ in vivo. To further investigate the above effects, we injected the human ovarian cancer $\mathrm{C} 13^{*}$ cells and primary human ovarian cancer-associated fibroblasts (CAFs) into athymic BALB/c nude mice. The tumor weight and tumor growth were assessed after DDP treatment for four weeks. As shown in Fig. 6A-C, the tumor weight and tumor volume of $\mathrm{C}^{*}{ }^{*}$ cells with CAFs in the right flank increased rapidly compared with $\mathrm{C} 13^{*}$ cells alone in the left flank. IHC assay indicated that the right tumors displayed an increased proliferation percentage of $\mathrm{Ki}-67$ positive tumor cells and decreased apoptosis rates of activated caspase-3 positive tumor cells. Tumors in the right flank exhibited increased CCL5 expression compared with left flank tumors. Moreover, expression of p-STAT3, and p-Akt were strongly increased in the group injected with $\mathrm{C} 13^{*}$ mixed with CAFs compared with the group injected with $\mathrm{C} 13^{*}$ alone after DDP treatment for four weeks (Fig. 6D). Taken together, these results demonstrated that CCL5 derived from CAFs promoted not only ovarian cancer cell resistance to DDP in vitro, but also ovarian cancer cell proliferation, growth and resistance to DDP in vivo.

\section{Discussion}

Cisplatin-based treatment is the first-line chemotherapy for ovarian cancer patients after tumor debulking surgery. However, acquired cisplatin resistance commonly results in treatment failure and high incidence of tumor relapse and mortality.
Therefore, better understanding of the mechanisms involved in the regulation of cisplatin resistance is crucial for improving ovarian cancer treatment. Many studies have focused on cellautonomous mechanisms of cisplatin resistance. In contrast, we propose that cisplatin-induced CCL5 secretion derived from the tumor microenvironment, whose dominant components are CAFs, confers acquired resistance to cisplatin. Recent studies have demonstrated that cells in the tumor microenvironment could modulate the response of cancer cells to chemotherapy through the production of secreted factors. Sun et al (2) found that treatment-induced damage to the tumor microenvironment promoted prostate cancer therapy resistance through WNT16B. Nuclear factor- $\kappa \mathrm{B}(\mathrm{NF}-\kappa \mathrm{B})$ is a key component in mediating WNT16B upregulation upon DNA damage caused by chemotherapy (38). Bruchard et al (39) argued that chemotherapy-triggered cathepsin B released in myeloidderived suppressor cells activates the N1rp3 inflammasome and promotes tumor growth, indicating a new mechanism of chemotherapy resistance. Two other studies have shown that paracrine signaling from tumor microenvironment cells can affect cancer cell drug sensitivity $(40,41)$.

In the tumor microenvironment, CAFs not only play crucial roles in tumor growth, progression, and metastasis, but are also involved in drug resistance. Here, we investigated the role of cisplatin-induced CCL5 secretion from CAFs in ovarian cancer cell resistance to cisplatin. CCL5, also known as RANTES, is a member of the CC-chemokine family and plays a critical role in tumor progression and prognosis (3). A previous study demonstrated that CCL5 could be used as a prognostic indicator for breast and cervical cancer (6). High plasma CCL5 levels were positively associated with advanced breast cancer, and tumor-cell derived CCL5 might promote 
breast cancer progression and metastasis (6). To the best of our knowledge, this is the first study to investigate whether CCL5 promotes ovarian cancer cell resistance to cisplatin in vitro and in vivo. Human recombination CCL5 neutralization antibody was used to verify whether CCL5 substantially promoted cisplatin resistance, and acquired cisplatin resistance was abolished in vitro. These results partly explain CCL5-induced cisplatin resistance. We focused on the STAT3 signaling pathway, a member of STAT transcription factor family, given its significant role in tumorigenesis and regulation of chemotherapy resistance in cancer cells $(17,18,42)$. Previous studies have demonstrated that STAT3 and CCL5 contribute to the maintenance of tamoxifen resistance in breast cancer, and STAT3 phosphorylation is constitutively activated and retained via CCL5 stimulation $(3,9,43)$. Based on these reports, we examined the signaling pathways activated by CCL5 in vitro and in vivo and found that STAT3 phosphorylation was enhanced in a time- and dose-dependent manner. We also found that AKT phosphorylation levels, which play a vital role in tumor malignancy phenotype, were significantly activated by CCL5 stimulation, consistent with previous reports $(19,33,34)$. Next, we used small molecular interfering RNA to knock down STAT3 gene expression or the small molecular inhibitor LY294002 to inhibit the PI3K-Akt signaling pathway. Acquired cisplatin resistance was significantly reversed via CCL5 stimulation, similar to administration of the CCL5neutralizing antibody. Expression levels of the anti-apoptotic gene Bcl-2 appeared to be positively associated with p-STAT3 levels, in agreement with a previous study, indicating that STAT3 activation plays a critical role in inhibiting apoptosis and promoting proliferation by regulating the Bcl-2 family (44-46).

Previous studies have demonstrated that tumor cells are the main driver of lymphangiogenesis and angiogenesis promotion by secreting specific factors $(47,48)$. Here, we focused on elements in the tumor microenvironments and preliminarily explored the mechanisms of cisplatin-induced CCL5 secretion derived from CAFs, which results in acquired resistance to cisplatin. The present study has several disadvantages. We confirmed cisplatin-induced CCL5 secretion may promote cisplatin resistance in ovarian cancer in vivo and in vitro but did not confirm whether other chemotherapy drugs or small molecular-targeted therapies have similar effects. Thus, further research in other tumor types investigating proper combination therapies in vivo should be conducted.

This study found, for the first time, that cisplatin-induced CCL5 secretion derived from CAFs was able to promote tumor progression and drug-resistance through the p-STAT3 and p-Akt signal pathways. Taken together, our results support several conclusions. First, we observed that cisplatin resistance occurs via the tumor microenvironment, not only the tumor cells, which more closely reflects the real body environment, providing a novel foundation for the mechanism of cisplatin resistance. Second, our results suggested that components of the tumor microenvironment are important contributors to acquired cisplatin resistance. Third, clinical data confirmed that CCL5 expression is strongly correlated with chemotherapy sensitivity, which may provide a reference for clinical drug use. Lastly, we demonstrated that combining approaches that target constituents of the tumor microenvi- ronment might enhance conventional cancer chemotherapy. Therefore, STAT3 knockdown, small molecule inhibitors, or CCL5 blockade using neutralizing antibody in combination with conventional cisplatin treatment may provide a new therapeutic approach for ovarian cancer patients with acquired cisplatin resistance.

\section{Acknowledgements}

The present study was supported by the National Basic Research Program of China (973 Program, 2015CB553903); the Chinese 863 Program (2012AA02A507); the National Nature and Science Foundation of China (81230038; 81272859; 81025011; 81090414; 81000979; 81101962).

\section{References}

1. Bast RC Jr: Molecular approaches to personalizing management of ovarian cancer. Ann Oncol 2 (Suppl 8): viii5-viiil, 2011.

2. Sun Y, Campisi J, Higano C, Beer TM, Porter P, Coleman I, True L and Nelson PS: Treatment-induced damage to the tumor microenvironment promotes prostate cancer therapy resistance through WNT16B. Nat Med 18: 1359-1368, 2012.

3. Yi EH, Lee CS, Lee JK, Lee YJ, Shin MK, Cho CH, Kang KW, Lee JW, Han W, Noh DY, et al: STAT3-RANTES autocrine signaling is essential for tamoxifen resistance in human breast cancer cells. Mol Cancer Res 11: 31-42, 2013.

4. Velasco-Velázquez M, Jiao X, De La Fuente M, Pestell TG, Ertel A, Lisanti MP and Pestell RG: CCR5 antagonist blocks metastasis of basal breast cancer cells. Cancer Res 72: 3839-3850, 2012.

5. Luboshits G, Shina S, Kaplan O, Engelberg S, Nass D, LifshitzMercer B, Chaitchik S, Keydar I and Ben-Baruch A: Elevated expression of the $\mathrm{CC}$ chemokine regulated on activation, normal $\mathrm{T}$ cell expressed and secreted (RANTES) in advanced breast carcinoma. Cancer Res 59: 4681-4687, 1999.

6. Niwa Y, Akamatsu H, Niwa H, Sumi H, Ozaki Y and Abe A: Correlation of tissue and plasma RANTES levels with disease course in patients with breast or cervical cancer. Clin Cancer Res 7: 285-289, 2001.

7. Zhang Y, Yao F, Yao X, Yi C, Tan C, Wei L and Sun S: Role of CCL5 in invasion, proliferation and proportion of $\mathrm{CD}_{4} 4^{+} /$ CD24 phenotype of MCF-7 cells and correlation of CCL5 and CCR5 expression with breast cancer progression. Oncol Rep 21: 1113-1121, 2009.

8. Jiao X, Katiyar S, Willmarth NE, Liu M, Ma X, Flomenberg N, Lisanti MP and Pestell RG: c-Jun induces mammary epithelial cellular invasion and breast cancer stem cell expansion. J Biol Chem 285: 8218-8226, 2010.

9. Karnoub AE, Dash AB, Vo AP, Sullivan A, Brooks MW, Bell GW, Richardson AL, Polyak K, Tubo R and Weinberg RA: Mesenchymal stem cells within tumour stroma promote breast cancer metastasis. Nature 449: 557-563, 2007.

10. Bromberg J and Darnell JE Jr: The role of STATs in transcriptional control and their impact on cellular function. Oncogene 19: 2468-2473, 2000.

11. Diaz N, Minton S, Cox C, Bowman T, Gritsko T, Garcia R, Eweis I, Wloch M, Livingston S, Seijo E, et al: Activation of stat 3 in primary tumors from high-risk breast cancer patients is associated with elevated levels of activated SRC and survivin expression. Clin Cancer Res 12: 20-28, 2006.

12. Johnston PA and Grandis JR: STAT3 signaling: Anticancer strategies and challenges. Mol Interv 11: 18-26, 2011.

13. Zhong Z, Wen Z and Darnell JE Jr: Stat3: A STAT family member activated by tyrosine phosphorylation in response to epidermal growth factor and interleukin-6. Science 264: 95-98, 1994.

14. Fukada T, Hibi M, Yamanaka Y, Takahashi-Tezuka M, Fujitani Y, Yamaguchi T, Nakajima $\mathrm{K}$ and Hirano T: Two signals are necessary for cell proliferation induced by a cytokine receptor gp130: Involvement of STAT3 in anti-apoptosis. Immunity 5: 449-460, 1996 
15. Bowman T, Garcia R, Turkson J and Jove R: STATs in oncogenesis. Oncogene 19: 2474-2488, 2000.

16. Kube D, Holtick U, Vockerodt M, Ahmadi T, Haier B, Behrmann I, Heinrich PC, Diehl V and Tesch H: STAT3 is constitutively activated in Hodgkin cell lines. Blood 98: 762-770, 2001.

17. Sheng WJ, Jiang H, Wu DL and Zheng JH: Early responses of the STAT3 pathway to platinum drugs are associated with cisplatin resistance in epithelial ovarian cancer. Braz J Med Biol Res 46: 650-658, 2013 .

18. Han Z, Feng J, Hong Z, Chen L, Li W, Liao S, Wang X, Ji T, Wang S, Ma D, et al: Silencing of the STAT3 signaling pathway reverses the inherent and induced chemoresistance of human ovarian cancer cells. Biochem Biophys Res Commun 435: 188-194, 2013

19. Huang CY, Fong YC, Lee CY, Chen MY, Tsai HC, Hsu HC and Tang CH: CCL5 increases lung cancer migration via PI3K, Akt and NF-kappaB pathways. Biochem Pharmacol 77: 794-803, 2009.

20. Asselin E, Mills GB and Tsang BK: XIAP regulates Akt activity and caspase-3-dependent cleavage during cisplatin-induced apoptosis in human ovarian epithelial cancer cells. Cancer Res 61: 1862-1868, 2001

21. Navab R, Strumpf D, Bandarchi B, Zhu CQ, Pintilie M, Ramnarine VR, Ibrahimov E, Radulovich N, Leung L, Barczyk M, et al: Prognostic gene-expression signature of carcinoma-associated fibroblasts in non-small cell lung cancer. Proc Natl Acad Sci USA 108: 7160-7165, 2011.

22. Weng D, Song X, Xing H, Ma X, Xia X, Weng Y, Zhou J, Xu G, Meng L, Zhu T, et al: Implication of the Akt2/survivin pathway as a critical target in paclitaxel treatment in human ovarian cancer cells. Cancer Lett 273: 257-265, 2009.

23. Sun C, Li N, Yang Z, Zhou B, He Y, Weng D, Fang Y, Wu P, Chen P, Yang X, et al: miR-9 regulation of BRCA1 and ovarian cancer sensitivity to cisplatin and PARP inhibition. J Natl Cancer Inst 105: 1750-1758, 2013.

24. Gottesman MM, Fojo T and Bates SE: Multidrug resistance in cancer: Role of ATP-dependent transporters. Nat Rev Cancer 2 48-58, 2002

25. Cole SP, Bhardwaj G, Gerlach JH, Mackie JE, Grant CE, Almquist KC, Stewart AJ, Kurz EU, Duncan AM and Deeley RG Overexpression of a transporter gene in a multidrug-resistant human lung cancer cell line. Science 258: 1650-1654, 1992

26. Kruh GD and Belinsky MG: The MRP family of drug efflux pumps. Oncogene 22: 7537-7552, 2003.

27. Seike T, Fujita K, Yamakawa Y, Kido MA, Takiguchi S, Teramoto N, Iguchi $\mathrm{H}$ and Noda $\mathrm{M}$ : Interaction between lung cancer cells and astrocytes via specific inflammatory cytokines in the microenvironment of brain metastasis. Clin Exp Metastasis 28: 13-25, 2011.

28. Wang D, Yamamoto S, Hijiya N, Benveniste EN and Gladson CL: Transcriptional regulation of the human osteopontin promoter: Functional analysis and DNA-protein interactions. Oncogene 19: 5801-5809, 2000

29. Rath BH, Fair JM, Jamal M, Camphausen K and Tofilon PJ: Astrocytes enhance the invasion potential of glioblastoma stem-like cells. PLoS One 8: e54752, 2013.

30. Liu L, Nam S, Tian Y, Yang F, Wu J, Wang Y, Scuto A, Polychronopoulos P, Magiatis P, Skaltsounis L, et al 6-Bromoindirubin-3'-oxime inhibits JAK/STAT3 signaling and induces apoptosis of human melanoma cells. Cancer Res 71 3972-3979, 2011.

31. Karar J and Maity A: PI3K/AKT/mTOR pathway in angiogenesis. Front Mol Neurosci 4: 51, 2011.

32. Zhang HY, Zhang PN and Sun H: Aberration of the PI3K/AKT/ mTOR signaling in epithelial ovarian cancer and its implication in cisplatin-based chemotherapy. Eur J Obstet Gynecol Reprod Biol 146: 81-86, 2009.
33. Tang $\mathrm{CH}$, Yamamoto A, Lin YT, Fong YC and Tan TW Involvement of matrix metalloproteinase-3 in CCL5/CCR5 pathway of chondrosarcomas metastasis. Biochem Pharmacol 79: 209-217, 2010

34. Wang SW, Wu HH, Liu SC, Wang PC, Ou WC, Chou WY, Shen YS and Tang CH: CCL5 and CCR5 interaction promotes cell motility in human osteosarcoma. PLoS One 7: e35101, 2012.

35. Eissa SA, Zaki SA, El-Maghraby SM and Kadry DY: Importance of serum IL-18 and RANTES as markers for breast carcinoma progression. J Egypt Natl Canc Inst 17: 51-55, 2005.

36. Kim HK, Song KS, Park YS, Kang YH, Lee YJ, Lee KR, Kim HK, Ryu KW, Bae JM and Kim S: Elevated levels of circulating platelet microparticles, VEGF, IL-6 and RANTES in patients with gastric cancer: possible role of a metastasis predictor. Eur J Cancer 39: 184-191, 2003.

37. Tsukishiro S, Suzumori N, Nishikawa H, Arakawa A and Suzumori K: Elevated serum RANTES levels in patients with ovarian cancer correlate with the extent of the disorder. Gynecol Oncol 102: 542-545, 2006.

38. Ostman A: The tumor microenvironment controls drug sensitivity. Nat Med 18: 1332-1334, 2012.

39. Bruchard M, Mignot G, Derangère V, Chalmin F, Chevriaux A Végran F, Boireau W, Simon B, Ryffel B, Connat JL, et al: Chemotherapy-triggered cathepsin $\mathrm{B}$ release in myeloid-derived suppressor cells activates the Nlrp3 inflammasome and promotes tumor growth. Nat Med 19: 57-64, 2013.

40. Wilson TR, Fridlyand J, Yan Y, Penuel E, Burton L, Chan E, Peng J, Lin E, Wang Y, Sosman J, et al: Widespread potential for growth-factor-driven resistance to anticancer kinase inhibitors. Nature 487: 505-509, 2012.

41. Straussman R, Morikawa T, Shee K, Barzily-Rokni M, Qian ZR, Du J, Davis A, Mongare MM, Gould J, Frederick DT, et al: Tumour micro-environment elicits innate resistance to RAF inhibitors through HGF secretion. Nature 487: 500-504, 2012.

42. Benabbou N, Mirshahi P, Cadillon M, Soria J, Therwath A and Mirshahi M: Hospicells promote upregulation of the ATP-binding cassette genes by insulin-like grow th factor-I via the JAK2/STAT3 signaling pathway in an ovarian cancer cell line. Int J Oncol 43: 685-694, 2013.

43. Kim JE, Kim HS, Shin YJ, Lee CS, Won C, Lee SA, Lee JW, Kim Y, Kang JS, Ye SK, et al: LYR71, a derivative of trimeric resveratrol, inhibits tumorigenesis by blocking STAT3-mediated matrix metalloproteinase 9 expression. Exp Mol Med 40: 514-522, 2008.

44. Ji T, Gong D, Han Z, Wei X, Yan Y, Ye F, Ding W, Wang J, Xia X, Li F, et al: Abrogation of constitutive Stat 3 activity circumvents cisplatin resistant ovarian cancer. Cancer Lett 341: 231-239, 2013.

45. Aoki Y, Feldman GM and Tosato G: Inhibition of STAT3 signaling induces apoptosis and decreases survivin expression in primary effusion lymphoma. Blood 101: 1535-1542, 2003.

46. Real PJ, Sierra A, De Juan A, Segovia JC, Lopez-Vega JM and Fernandez-Luna JL: Resistance to chemotherapy via Stat3dependent overexpression of $\mathrm{Bcl}-2$ in metastatic breast cancer cells. Oncogene 21: 7611-7618, 2002.

47. Cao Y: Opinion: Emerging mechanisms of tumour lymphangiogenesis and lymphatic metastasis. Nat Rev Cancer 5: 735-743, 2005.

48. Rasila KK, Burger RA, Smith H, Lee FC and Verschraegen C: Angiogenesis in gynecological oncology-mechanism of tumor progression and therapeutic targets. Int J Gynecol Cancer 15: $710-726,2005$ 